\title{
The role of dapsone in refractory idiopathic granulomatous mastitis
}

\author{
Ayla Pelleg, Kirangowda Pombarahalli Varadaraju, Swati Datta \\ John H Stroger Jr. Hospital of Cook County, Department of Internal Medicine, Chicago, IL, USA
}

Received: November 7, 2016

DOI: $10.5430 /$ crim.v4n $1 \mathrm{p} 72$
Accepted: January 17, $2017 \quad$ Online Published: February 5, 2017

URL: https://doi.org/10.5430/crim.v4n1p72

\begin{abstract}
Idiopathic Granulomatous Mastitis (IGM) is a benign inflammatory breast disease. A definite etiology of IGM is still unknown. IGM classically presents as an unilateral, painful breast mass in women who are 30-40 years of age. Consensus treatment guidelines do not exist for the management of IGM. In this case report, we present a patient with IGM who was refractory to Prednisone and started on Dapsone as an alternative treatment. This case report highlights the use of Dapsone and the need for further research of IGM treatment modalities.
\end{abstract}

Key Words: Granulomatous breast disease, Breast mass, Prednisone, Dapsone, Refractory

\section{BACKGROUND}

Idiopathic Granulomatous Mastitis (IGM) is a benign inflammatory breast disease first described in 1972. A definite etiology of IGM is still unknown. Possible etiologies may involve infectious or inflammatory processes. However, isolation of an organism has proved difficult and a target for an autoimmune trigger is still unknown. ${ }^{[1]}$ Environmental and genetic factors may also play a role in the pathogenesis of IGM. ${ }^{[2]}$

IGM classically presents as a unilateral painful breast mass in women who are 30-40 years of age. ${ }^{[1]}$ When first encountered, IGM may mimic infectious mastitis or malignancy. Correct diagnosis depends on histopathologic findings of non-caeseating granulomas around the breast lobules. Idiopathic granulomatous mastitis should be differentiated from other granulomatous diseases such as Wegener's granuloma, sarcoidosis, tuberculosis and histoplasmosis.

Consensus treatment guidelines do not exist for the manage- ment of IGM. Common IGM treatments include: conservative therapy, oral steroids and surgery. Recurrences may occur in all treatment modalities. Immunomodulators such as Azathiprine and Methotrexate as well as surgery have also been used in patients with more refractory cases. ${ }^{[1,2]}$ Recurrences can happen in all treatment modalities.

\section{CASE DeSCRIPTION}

A 33-year-old African American female presented with a two week, right-sided painful breast lump. Physical examination revealed the right breast to be larger than the left breast, with minimal erythema, tenderness to palpation and an $11 \mathrm{~cm} \times 6 \mathrm{~cm}$ palpable mass in the upper right breast quadrant. She had been treated with antibiotics for one week with no improvement.

Thus, a core biopsy of the breast lesion was performed. AFB stain and culture were negative; however, the initial biopsy culture grew Actinomyces Odontolyticus. Pathology

${ }^{*}$ Correspondence: Ayla Pelleg; Email: apelleg@ cookcountyhhs.org; Address: John H Stroger Jr. Hospital of Cook County, Department of Internal Medicine, Chicago, IL, USA. 
revealed non-necrotizing granulomas, granulation tissue and fibrosis (see Figures 1, 2). After consultation with Infectious Disease, the patient was started on Amoxicillin $500 \mathrm{mg}$ daily.
After one month, the patient continued to have significant breast pain. It was questionable if a pure infectious process was present; thus, Prednisone $60 \mathrm{mg}$ daily was started.

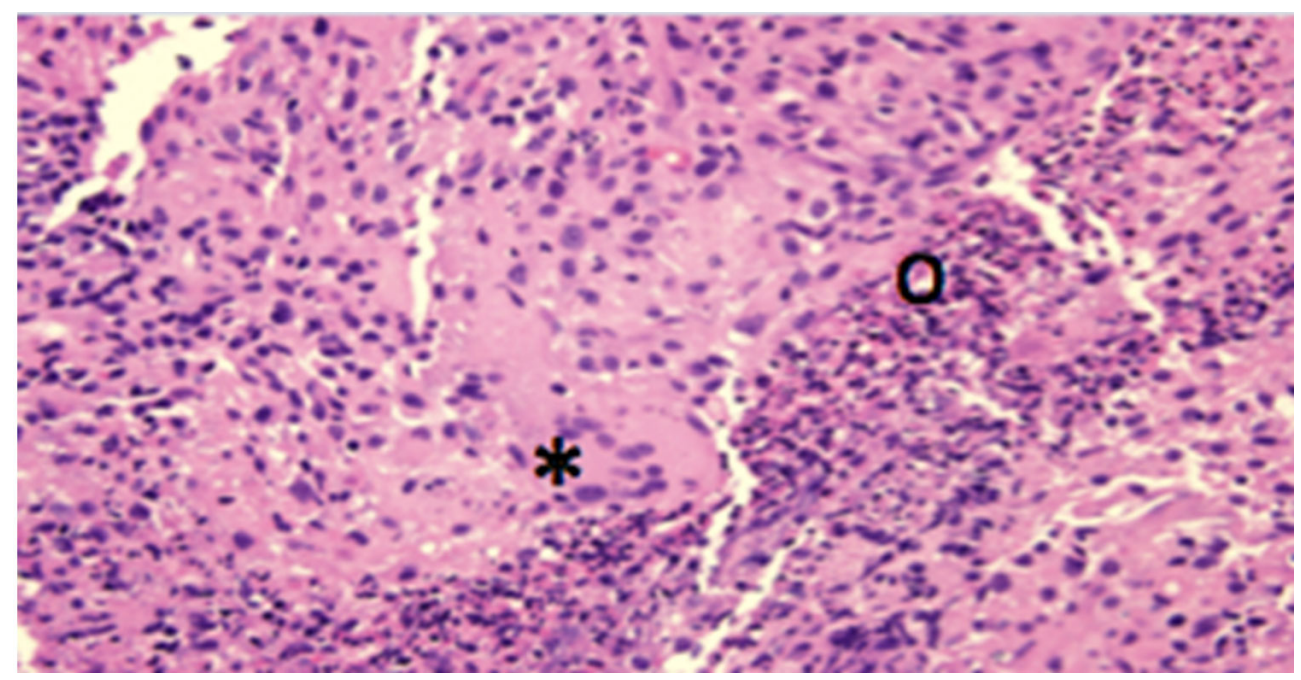

Figure 1. Defined granuloma with multinucleated giant cell (*) and acute suppurative exudate (o), Hematoxylin and eosin stain; $40 \times$

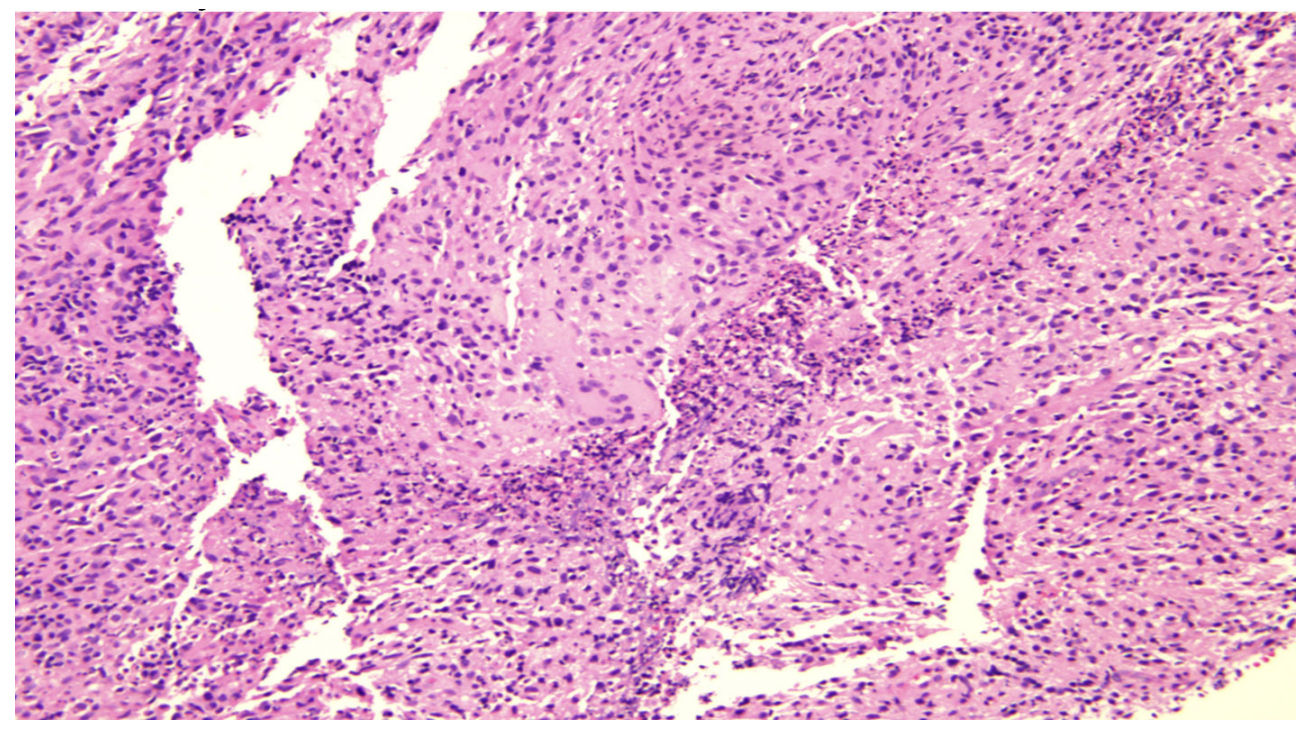

Figure 2. Acute and chronic mastitis with ill-defined granuloma, non-necrotizing, Hematoxylin and eosin stain; 20×

After six months of a prolonged course of Prednisone with multiple failed attempts to taper, the patient was offered steroid-sparing agents. The patient declined starting Methotrexate and Azathioprine due to concerns of possible side effects. After consultation with Dermatology, an alternative treatment with Dapsone was offered. The patient was initially started on Dapsone $25 \mathrm{mg}$ daily and slowly increased to $100 \mathrm{mg}$ daily over three months. The patient improved while on Dapsone and was finally tapered off prednisone six months after starting Dapsone (see Figure 3).

\section{Discussion}

Based on a comprehensive review of literature, Dapsone has not been previously used in the direct treatment of IGM. Dapsone's use as an antibiotic is well understood; acting as a competitive antagonist of Para Amino Benzoic Acid (PABA) in the folate synthesis pathway. Dapsone has also been used as an anti-inflammatory agent. However, Dapsone's anti-inflammatory mechanism of action is not clear. In vitro studies suggest that Dapsone inhibits the expression of Beta 2 integrin (CD11/CD18) mediated adhesion of neu- 
trophils, interfering in the inflammatory pathway. ${ }^{[3]}$ Dapsone has been used to treat Dermatitis Herpetiformis (DH) and Sweet Syndrome (SS). Histopathology of these lesions usu- ally shows significant neutrophil infiltrates, which may help explain Dapsone's efficacy in both DH and SS.
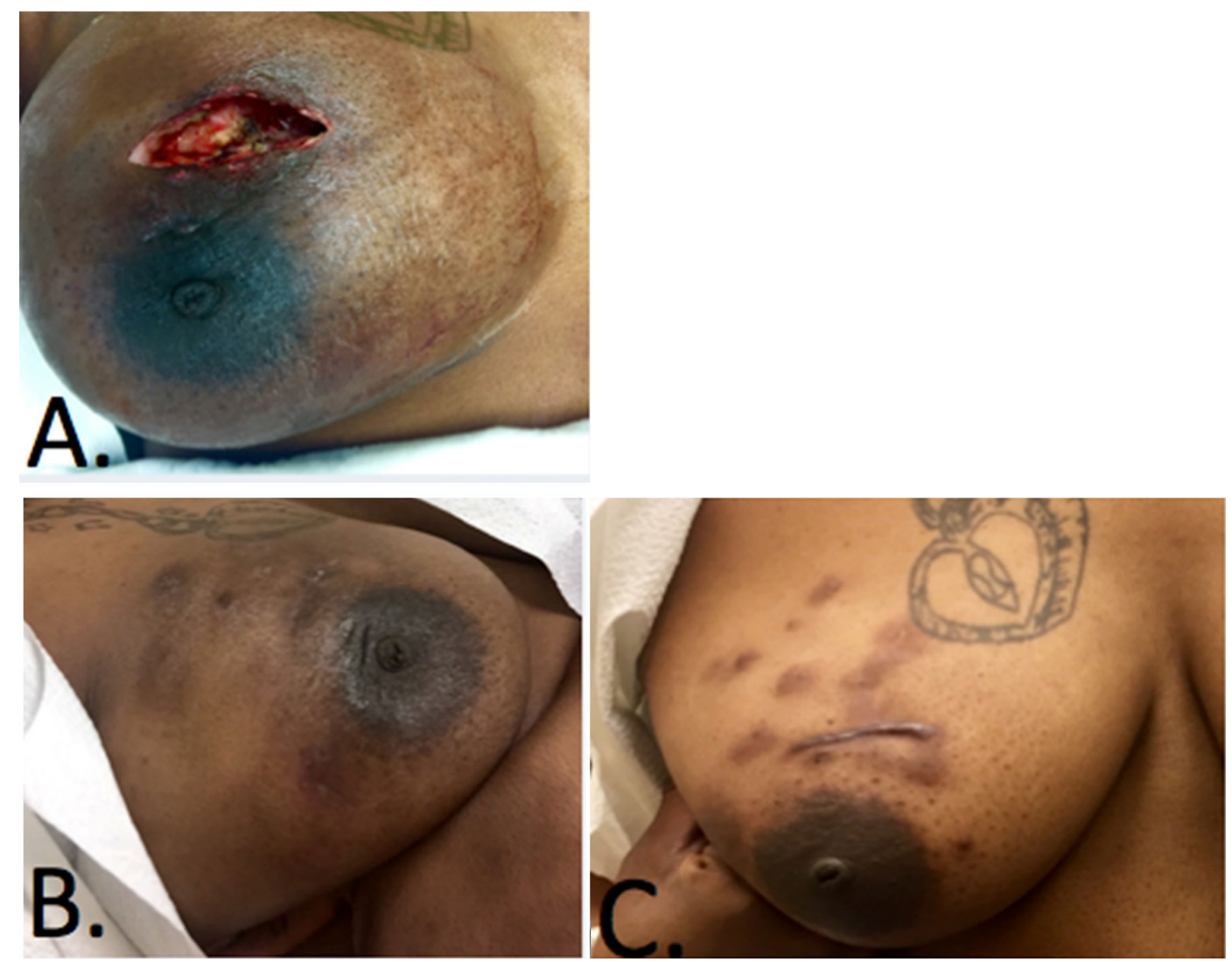

Figure 3. A. Right breast after surgical biopsy; B. Right breast after the start of Prednisone with continued, active granulomatous lesions; C. Right breast after the start of Dapsone with mild, active granulomatous lesions

Review of pathology slides from this patient with IGM revealed non-necrotizing granulomas along with significant neutrophilic infiltration. Considering the in vitro mechanism of action on neutrophils, Dapsone was offered as treatment to this patient.

Although this patient clinically improved while on Dapsone, it is difficult to discern if this was directly due to Dapsone, or if this was the natural course of the disease. Further research on diagnostic modalities and treatment for refractory IGM cases is needed to better understand treatment mechanism of actions and formulate consensus treatment guidelines.

\section{Conflicts of Interest Disclosure}

The authors have no competing interests to declare.

\section{REFERENCES}

[1] Shebani F, Sarvghad M, Naderi HR, et al. Treatment for and clinical characteristics of granulomatous mastitis. Obstetrics and Gynecology. 2015; 125(4): 801-7. PMid:25751209

[2] Pandey TS, Mackinnon JC, Millar A, et al. Idiopathic granuloma- tous mastitis - a prospective study of 49 women and treatment outcomes with steroid therapy. Breast Journal. 2014; 20(3): 258-66. https://doi.org/10.1097/A0G.0000000000000734

[3] Wozel G, Blasum C. Dapsone in dermatology and beyond. Archives of Dermatological Research. 2014; 306(2): 103-24. 